\title{
Clinical Outcome and Survival of Post-molar GTN Versus Non-molar GTN Patients
}

\author{
Shina Oranratanaphan, Ruangsak Lertkhachonsuk
}

Department of Obstetrics and Gynecology Faculty of Medicine Chulalongkorn University, Bangkok, Thailand.

\begin{abstract}
Background: Gestational trophoblastic neoplasia (GTN) can derive either from molar or non-molar pregnancy. Our primary objective is to compare clinical presentation and outcome of treatment of non-molar and post-molar GTN. Our secondary outcome is to evaluate and compare the prognostic factors of non-molar GTN compare to post-molar GTN in subgroup classification of GTN patients by stage and by low-risk and high-risk groups. Methods: Retrospective chart review of GTN patients treated from 2007 to 2016 was done. General characteristics, clinical data, treatment options, and treatment outcomes were collected. The cases with missing significant data were excluded. Statistics analysis of the data was performed with SPSS version 22.0. Mean, mode, median and percent were used to present the data. Student t-test, Mann Whitney-U test and Kaplan Meier were used to analyze the data. The results were presented in Tables or graphs where appropriate. Results: Total of 71 GTN patients was recruited into the study. Fifty-one patients were post-molar GTN and 20 were non-molar GTN patients. The mean age of the patients was not different $(\mathrm{p}=0.25)$. Median duration from previous pregnancy and time to achieve remission were longer in non-molar GTN (292 days vs. 42 days and 163 vs. 64 days, respectively). Mortality rate of the non-molar GTN is higher that of the post-molar GTN (15\% vs. 1.9\%). Comparison of stage to stage showed no differences between the post-molar and the non-molar GTN. According to previous pregnancy type, post-abortion had higher resistant to treatment rate than post-term delivery. Conclusion: Non-molar GTN is different form post-molar GTN in several aspects, such as the duration from previous pregnancy, stage and score at diagnosis, treatment resistance and mortality rate. Comparison between the non-molar and post-molar GTN stage by stage and risk scores could not identify the difference between the two groups.
\end{abstract}

Keywords: Non-molar GTN- post-molar GTN- survival- clinical outcome- prognosis

Asian Pac J Cancer Care, 4 (4), 113-118

\section{Introduction}

Gestational trophoblastic disease (GTD) is a group of diseases which comprises both benign and malignant disorders [1]. The malignant form of GTD is called gestational trophoblastic neoplasia (GTN) [2]. Incidence of GTD and GTN varies worldwide. The incidence in Asia is higher than that in Europe and America [3]. For example, the overall incidence is estimated at 1 per 50,000 pregnancies worldwide. Whereas the incidence in the USA is 1-2 per 100,000 pregnancies, the incidence in China is 202 per 100,000 pregnancies [4].

GTN can derive from both molar and non-molar pregnancy. GTN derived from molar pregnancy is called post-molar GTN and GTN derived from non-molar pregnancy such as term delivery, abortion or ectopic
Submission Date: 04/08/2019 Acceptance Date: 07/06/2019

pregnancy is called non-molar GTN. Fifty percent of GTN derived from molar pregnancy while $20-25 \%$ derived from term delivery and $25-30 \%$ derived from ectopic pregnancy or abortion [5-6]. Post-molar and non-molar GTN are quite different in many aspects. Post-molar GTN is most commonly diagnosed during follow-up $\beta$-hCG titer after termination of molar pregnancy according to FIGO criteria for diagnosis of post-molar GTN [7].

According to the diagnostic criteria and regular follow up of highly sensitive tumor marker such as $\beta$ hCG, post-molar GTN is usually diagnosed at early stage. On the other hand, non-molar GTN is usually diagnosed at late stage and has longer duration from previous pregnancy because of the lack of follow up and the patients usually presented with the metastatic symptoms such as cough or hemoptysis from lung metastasis or headache or seizure or

Corresponding Author

Dr. Shina Oranratanaphan

Department of Obstetrics and Gynecology Faculty of Medicine Chulalongkorn University, Bangkok, Thailand.

Email: dr_shina@hotmail.com 
intracranial hemorrhage from brain metastasis. Therefore, non-molar GTN is considered as a poor prognostic factor of GTN.

Severity of GTN is depended on risk classification from FIGO staging and FIGO scoring system (International Federation of Gynecologic and Obstetrics (2000) scoring system for gestational trophoblastic neoplasia). The score 0-6 is considered as low-risk which stands for low-risk of resistance to chemotherapy. Most of the GTN patients (95\%) are low-risk patients which are recommended to start treatment with single agent chemotherapy [8]. The patients whose score are 7 or more are considered as high-risk patients and the treatment should begin with multiagent chemotherapy. Moreover, the cases that score are 13 or more is considered as extremely high-risk patients which sophisticated treatments are required. Overall response rate for treatment of GTN patients was $50-90 \%$. Cure rate of low-risk patients is high as $99-100 \%$ and cure rate for high-risk patients are 60-90\% [8-9]. Non-molar GTN patients usually present at late stage and high score. Therefore, many works of literature considered non molar GTN as high-risk for chemo-resistance and poor prognosis predictor [10-11]. Other poor prognostic factors for GTN are stage of the disease, duration from previous pregnancy, pretreatment $\beta$-hCG level, history of failure of treatment with chemotherapy and liver metastasis [10-12].

When considering those risk factors, many factors are overlapped in clinical presentation of non-molar GTN. This study was conducted to evaluate the clinical presentation of non-molar GTN compare to Post-molar GTN. Our primary objective is to compare clinical presentation and treatment outcome of non-molar GTN and post-molar GTN. Our secondary outcome is to evaluate and compare non-molar GTN compare to post-molar GTN as subgroup classification of GTN patient by stage and by low-risk and high-risk groups.

\section{Materials and Methods}

Retrospective study was performed after Ethics committee approved the protocol. The data of GTN patients who were treated at King Chulalongkorn Memorial Hospital (KCMH) from 2007 to 2016 were recruited. The cases that missed significant data were excluded from the study. The previous pregnancy was reviewed and pathological results of previous pregnancy were confirmed in case that the block slides were available. The diagnosis of GTN was reviewed. The stage and score were recalculated to confirm the data.

General characteristics, clinical data, treatment information and treatment outcome were collected. General characteristics data and clinical data included age, previous pregnancy, stage, score, presenting symptoms, duration from previous pregnancy and pretreatment $\beta$ hCG level. Treatment data included first line treatment, regimen of chemotherapy, number of cycles of chemotherapy used to achieve remission, adjuvant treatment and time to achieve complete remission. Treatment outcomes included results of first line treatment which were classified in response or resistance. Relapse of the disease during follow up time was collected. Final treatment results were classified as survive or die. The patients were followed up until December 2018 which meant follow up time of every patient in this cohort is more than 24 months.

SPSS version 22 was used to analyze the data. General characteristics were presented in mean, median or percentage as appropriate. T test, Chi-square and Mann Whitney-U test were used to compare between groups. Time to achieve remission was plotted in the graph with Kaplan Meier test.

\section{Results}

Retrospective chart review of the patients diagnoses GTN during the year 2007 and 2016 was performed. Seventy-one patients of GTN were recruited. Fifty-one patients were post-molar GTN and 20 patients were non-molar GTN. Most of post-molar GTN developed after complete hydatidiform mole. Only 5 from 51 patients developed after partial mole. None of PHM developed high-risk GTN. In non-molar GTN, 12 from 20 patients developed GTN after abortion and 8 from 20 patients developed GTN after delivered of term pregnancy. Mean age of post-molar GTN was 29.71 years and mean age of non-molar GTN was 32.65 years $(\mathrm{p}=0.25)$. The data about FIGO stage, risk, pre-treatment $\beta$-hCG level, duration from previous pregnancy and time to achieve remission are shown in Table 1. Median duration from previous pregnancy is significant longer in non-molar GTN (median 292 days VS 42 days; $p=0.00$ ). Median time to achieve remission in non-molar GTN was significant longer than post-molar GTN (163 vs. 64 days; $p=0.00)$. Most of post-molar GTN was stage 1 (43 from $51 ; 84.31 \%$ ) but most of non-molar GTN was stage 3 ( 8 from $20 ; 40 \%$ ). Likewise, most of post-molar GTN was low-risk ( 47 from 51; 92.16\%) but most of non-molar GTN was high-risk (18 from 20; 90\%). Post-molar GTN usually found without symptom $(20 / 51 ; 39.22 \%)$. The most common presenting symptoms in post-molar GTN group was vaginal bleeding $(24 / 51 ; 47.06 \%)$ while 16 from 20 patients of non-molar GTN presented with symptoms from metastasis such as vaginal bleeding or neurological symptoms. Most of post-molar GTN did not have distant metastasis (44/51; $86.27 \%$ ) only 2 patients had brain metastasis. Only 5 from 20 patients in non-molar GTN had no metastasis.

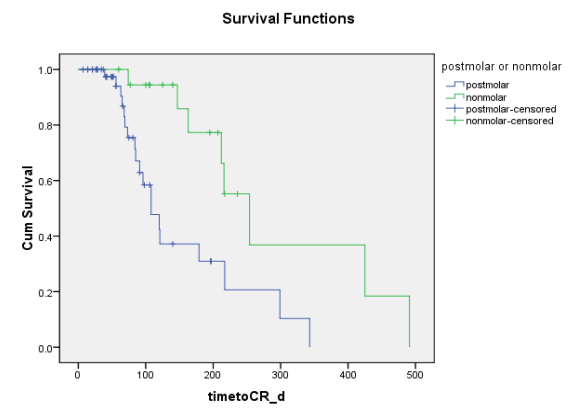

Figure 1. Comparison of Time to Achieve Remission in Post-molar and Non-molar GTN 
Table 1. General Characteristics of Post-molar GTN and Non-molar GTN Patients

\begin{tabular}{|c|c|c|c|}
\hline Character & Post-molar GTN & Non -molar GTN & $\mathrm{P}$ value \\
\hline Age $($ Mean + SD) & $29.71+9.30$ & $32.65+10.52$ & 0.252 \\
\hline Score ( Median (IQR)) & $2(1-4)$ & $11(7.5-13.0)$ & 0.00 \\
\hline Pretreatment hCG (Median (IQR) & $124402(21189.5-413526.25)$ & $213884.5(52332.25-678885.25)$ & 0.29 \\
\hline Duration from previous pregnancy (Median (IQR)) & $42(28-80.25)$ & $292(100.5-934.0)$ & 0.00 \\
\hline Time to achieve remission (Median (IQR)) & $64(38.4-100)$ & $163(105-217)$ & 0.00 \\
\hline \multicolumn{4}{|l|}{ Stage (N (\%)) } \\
\hline I & $43(84.31)$ & $5(25)$ & \\
\hline II & $2(3.92)$ & $2(10)$ & \\
\hline III & $4(7.84)$ & $8(40)$ & \\
\hline IV & $2(3.92)$ & $5(25)$ & \\
\hline \multicolumn{4}{|l|}{ Risk score (N (\%)) } \\
\hline Low-risk (score $0-6$ ) & $47(92.16)$ & $2(10)$ & \\
\hline High-risk & $4(7.84)$ & $18(90)$ & \\
\hline \multicolumn{4}{|l|}{ (score 7 or more) } \\
\hline Extremely High-risk & $2(3.92)$ & $6(30)$ & \\
\hline \multicolumn{4}{|l|}{ (Score 13 or more) } \\
\hline Resistance (N (\%)) & $13 / 51(25.49)$ & $6 / 20(30)$ & \\
\hline Relapse (N (\%)) & 6/51 (11.76) & $3 / 20(15)$ & \\
\hline Death (N (\%)) & $1 / 51(1.96)$ & $3 / 20(15)$ & \\
\hline
\end{tabular}

Lung metastasis was found in 7 from 10 patients $(35 \%)$. Brain metastasis was found in 6 from 20 patients $(30 \%)$. Brain metastasis without lung metastasis was found in 2 from 20 patients $(10 \%)$ which was one-third of all brain metastasis in this group.

Majority of treatment of both groups were chemotherapy. The most commonly used first line chemotherapy in post molar GTN was single agent chemotherapy such as Methotrexate $(25 / 51 ; 49.02 \%)$ followed with Acitnomycin D (21/51; 41.18\%). In non-molar GTN 9 from 20 patients (45\%) started with EMACO and $20 \%$ of the patients (4/20) started with EP (etoposide with cisplatin). Second line treatment was used in both groups $(19 / 51 ; 37.25 \%$ in post molar GTN and $7 / 20 ; 35 \%$ of non-molar GTN group). Resistance and relapse were found in both groups. Post-molar GTN patients developed resistance or relapse 19 cases form 51 cases $(37.25 \%)$. Non-molar GTN developed resistance or relapse 9 from 20 cases (45\%). In our study, $31 \%$ (16 from 49) of low-risk group of postmolar GTN resisted first line treatment but 100\% (2 from 2) of low-risk group of nonmolar GTN resisted to first line single agent treatment. As for the high-risk group, 75\% (3 from 4 cases) of post-molar GTN and 7 from 18 cases (38.89\%) of non-molar GTN resisted to first-line multiagent chemotherapy. Response rate in post-molar GTN and non-molar GTN were evaluated. As for post-molar GTN, overall response rate was $62.74 \%$, stage I was $62.79 \%$, stage II was $50 \%$ stage III was $75 \%$ and stage IV was $50 \%$. In low-risk post-molar GTN response rate was $65.96 \%$ and high-risk group was $25.3 \%$. As for the non-molar GTN, overall response rate was $55 \%$, stage I was $40 \%$, stage II was $0 \%$, stage III was $75 \%$ stage IV was $60 \%$, low-risk was $0 \%$ and high-risk was $61.11 \%$. CNS involvement on our study was 2 from 51 cases of post-molar GTN and 5 from 20cases (25\%) of non-molar GTN. Mortality was also found in both groups. Three patients in non-molar GTN died (15\%) while only 1 patient from the total of 51 patients in post-molar GTN died $(1.9 \%)$.

Subgroup analysis was performed. We stratified

Table 2. Data Comparing Low-risk and High-risk of Post-molar GTN and Non-molar GTN Patients

\begin{tabular}{|c|c|c|c|c|c|c|}
\hline \multirow[t]{2}{*}{ Factors } & \multicolumn{3}{|c|}{ Low-risk } & \multicolumn{3}{|c|}{ High-risk } \\
\hline & Post-molar GTN & Non-molar GTN & $P$ value & Post-molar GTN & Non-molar GTN & $P$ value \\
\hline $\begin{array}{l}\text { Duration from previous pregnancy } \\
(\text { Median }(\mathrm{IQR}))\end{array}$ & $42(28-71)$ & $240(120-310)$ & 0.234 & $318(145.25-1011.25)$ & $922(93.5-128.2)$ & 0.531 \\
\hline Pretreatment hCG & $\begin{array}{c}100097 \\
(19330.75-360243.75)\end{array}$ & 3108.5 & 0.1 & $\begin{array}{c}702377.5 \\
(286858.75-1014737.51)\end{array}$ & $\begin{array}{c}264702 \\
(76640.5-769578.25)\end{array}$ & 0.2 \\
\hline (Median (IQR)) & & $(2897-3338)$ & & & & \\
\hline $\begin{array}{l}\text { Cycle of chemotherapy } \\
(\text { Mean+SD) }\end{array}$ & $4.83+2.87$ & $8.50+0.71$ & 0.195 & $11.5+5.45$ & $8.74+5.59$ & 0.647 \\
\hline $\begin{array}{l}\text { Time to achieve remission } \\
(\text { Mean+SD) }\end{array}$ & $73.65+58.34$ & $110.5+51.62$ & 0.859 & $172.5+127.65$ & $195.82+115.43$ & 0.709 \\
\hline
\end{tabular}


Table 3. Comparison between Post-abortion and Post Term Delivery in Non-molar GTN Group

\begin{tabular}{|c|c|c|c|}
\hline Characteristics & Post-abortion $(n=12)$ & Post term delivery $(\mathrm{n}=8)$ & $\mathrm{P}$ value \\
\hline Age $(y r)($ mean $+\mathrm{SD})$ & $30.08+11.36$ & $36.50+8.32$ & 0.50 \\
\hline FIGO score (mean+ SD) & $10.08+4.12$ & $11.88+4.85$ & 0.92 \\
\hline Pre-pregnancy duration (days) (mean+ SD) & $958.36+2020.34$ & $1726.57+2401.08$ & 0.30 \\
\hline $\begin{array}{l}\text { Pretreatment hCG }(\mathrm{mIU} / \mathrm{ml}) \\
(\text { mean }+\mathrm{SD})\end{array}$ & $545596.47+879747.75$ & $468417.62+566666.12$ & 0.59 \\
\hline $\begin{array}{l}\text { Time to CR ( days) } \\
\text { (mean }+\mathrm{SD})\end{array}$ & $212.83+126.48$ & $142.29+71.74$ & 0.45 \\
\hline $\begin{array}{l}\text { Total cycles } \\
(\text { mean }+\mathrm{SD})\end{array}$ & $11.50+3.56$ & $5.00+5.20$ & 0.51 \\
\hline Number of first line cycle (mean + SD) & $7.83+4.11$ & $5.00+5.20$ & 0.59 \\
\hline $\begin{array}{l}\text { Number of second line } \\
(\text { mean }+\mathrm{SD})\end{array}$ & $3.33+3.87$ & $0.00+0.00$ & 0.01 \\
\hline Stage I (n (\%)) & $3(25)$ & $2(25)$ & 0.64 \\
\hline Stage II & $2(16.67)$ & $0(0)$ & \\
\hline Stage III & $4(33.33)$ & $4(50)$ & \\
\hline Stage IV & $3(25)$ & $2(25)$ & \\
\hline Low-risk & $2(16.67)$ & $0(0)$ & 0.22 \\
\hline High-risk & $10(83.33)$ & $8(100)$ & \\
\hline Response & $4(33.33)$ & 7 (87.5) & 0.02 \\
\hline Resistance & $8(66.67)$ & $1(12.5)$ & \\
\hline Cure & $10(83.33)$ & $7(87.5)$ & 0.8 \\
\hline Death & $2(16.67)$ & $1(12.5)$ & \\
\hline
\end{tabular}

post-molar and non-molar GTN patients in to stage and risk stratification. Duration from previous pregnancy, pretreatment $\beta$-hCG level and time to achieve remission were compared between post-molar and non-molar GTN in the same stage and same risk stratification. The results were shown in Table 2. Time to achieve remission was plotted in the graph and showed in Figure 1. Time to achieve remission, duration form previous pregnancy showed no difference between groups in subgroup analysis. Data on response of treatment and final response did not show any significant difference. Resistance to first line treatment was found in both low-risk and high-risk groups of either post-molar or non-molar GTN. Mortality cases were found in high-risk patients in both post-molar and non-molar GTN $(1-51 ; 1.96 \%$ and 3/20; $15 \%$, respectively). All of the low-risk patients survived.

Comparing previous pregnancy type in non-molar GTN group, post abortion had higher rate of resistant to first line treatment than post-term delivery. Details of comparison between post-abortion and post term delivery are shown in Table 3.

\section{Discussion}

GTN is malignant form of GTD which derived from either molar or non-molar pregnancy. Type of previous pregnancy is believed to be an important prognostic factor for response of treatment and treatment outcomes of GTN [10-11].

From the result of our study, non-molar GTN had long time to achieve remission and higher mortality rate compared to post-molar GTN. The mean age of both groups was not different.

Most of post-molar GTN had no symptom at the diagnosis and the diagnosis depended on hCG criteria which was different from non-molar GTN. Non-molar GTN patients mostly have symptoms at the diagnosis and the symptoms were associated with metastatic sites. Therefore, post-molar GTN patients were mostly diagnosed at earlier time and lower stage than non-molar GTN. For that reason, non-molar GTN in this study and in the previous studies were diagnosed in advanced stage and high scores and most of them were of high-risk for treatment failure [13]. Moreover, non-molar GTN in our study showed higher mortality rate, higher chance to resistance to first line chemotherapy and longer time to achieve remission which were poorer prognosis than post-molar GTN group. However, most of the nonmolar GTN cases were high-risk with high score while majority of the post-molar GTN group were stage 1 and low-risk patients. Moreover, non-molar GTN group had higher percentage of extremely high-risk patients that was known as the poorest prognosis factor. Besides nonmolar GTN, many factors such as anatomical stage, serum $\mathrm{hCG}$, duration from previous pregnancy, liver and brain metastasis and resistance to previous chemotherapeutic treatment were considered as poor prognostic factors [11-14]. Resistance to first line treatment is considered as poor prognostic factor for survival. From previous studies, $16-20 \%$ of low-risk GTN resisted first line 
treatment (single agent chemotherapy) and $20-30 \%$ of high-risk GTN resisted multiagent chemotherapy [12-15]. Our study found that $100 \%$ of low-risk non-molar GTN resisted to first line treatment (single agent chemotherapy) which was correlated to the guideline from IGR (Institut Gustave Roussy) [16] that advised to starting treatment with multiagent chemotherapy should be considered in the cases with pathological report was choriocarcinoma, non-molar GTN or metastatic sites were more than 10 lesions. Our resistance rate is higher than previous reports may be due to the high proportion of extremely high-risk cases. Extremely high-risk cases in our study are 50 $\%$ in post-molar GTN ( 2 from 4 cases) and $33.33 \%$ in non-molar GTN (6 from 18 cases). This can explain the reason for higher resistant rate in our study. Duration from antecedent pregnancy in non-molar GTN is longer than post-molar GTN. However, comparing post-molar and non-molar GTN in low-risk group cannot identify the difference, so did the high-risk group. Mortality rate was found in both groups. One case of post-molar GTN and 3 cases of non-molar GTN died. All of them belonged to the high-risk group. Fifty percent of the death cases were extremely high-risk. From previous studies, 5-year mortality increased in the GTN patients whose scores were more than 13 [17-18]. Response rate of treatment from previous studies were different by stage. Overall response rate was $66.7 \%$. The response rate in stage I and II were $100 \%$; stage III was $72.3 \%$ and stage IV were $38.5 \%$ [19]. Long-term remission was $73 \%$. Considering the response rates between post-molar and non-molar GTN stage by stage, the response rates in stage III and IV were quite similar; however, in stage I and II, the response rate of non-molar GTN was worse than post-molar GTN. CNS involvements in our study were more frequently found in non-molar GTN. CNS involvement rate was compatible with previous study that found CNS involvement $20 \%$ in non-molar GTN [20].

The score in FIGO scoring system includes previous pregnancy as a part of score. Therefore, non-molar pregnancy tended to have higher score than post-molar pregnancy. Moreover, post-abortion and post-term delivery had different score in FIGO scoring system. Post-term delivery had higher scores that may represent poorer prognosis [21]. However, our study found that post-abortion had higher resistant rate to first-line chemotherapy. These findings are correlated with the finding of Jiang et al. [22]. They found that post-abortion had higher hazard ratio than post-term delivery (2.78 vs. 2.27).

From the result of the study, many clinical manifestation and treatment outcome of post-molar GTN were not different from non-molar GTN but some outcome such as resistant to single agent chemotherapy and CNS involvement is more common in non-molar GTN. However, our study still had some limitations because of the retrospective design. GTN is a rare disease. This cohort contained 71 GTN patients who were still adherent with the protocol that could reassure at least 24 months of follow-up time in each patient.

However, our 10-year data had small number of high-risk patient in post-molar GTN patients ( 4 from 51 patients) and only 2 from 20 patients in non-molar GTN were of low-risk. Therefore, comparison of statistically significant interpretation between those groups may be limited. To answering the question that does non-molar GTN worse by itself or worse because of higher stage and score, the larger cohort with specified population may require

In conclusion, the overall non-molar GTN has poorer prognosis than post-molar GTN in many aspects such as longer time to achieve remission, higher risk of resistance to first-line treatment and mortality rate. However, within the same stage and risk classification, our study cannot detect the difference in time to achieve remission between groups. Further investigations are still required, as the population sizes in some groups were too small such as low-risk GTN in non-molar GTN group and high-risk GTN in post-molar GTN group. In non-molar GTN group, post-abortion had higher risk of resistance to first line treatment than post-term delivery.

\section{References}

1. Lurain JR. Gestational trophoblastic disease I: epidemiology, pathology, clinical presentation and diagnosis of gestational trophoblastic disease, and management of hydatidiform mole. Am J Obstet Gynecol 2010; 203(6): 531-9

2. Bagshawe KD, Dent J, Webb J. Hydatidiform mole in England and Wales 1973-83. Lancet 1986; 2(8508): 673-7.

3. Newlands ES, Paradinas FJ, Fisher RA. Recent advances in gestational trophoblastic disease. Hematol Oncol Clin North Am. 1999 Feb; 13(1):225-44.

4. Altieri A, Franceschi S, Ferlay J, Smith J, La Vecchia C. Epidemiology and aetiology of gestational trophoblastic diseases. Lancet Oncol 2003; 4 (11): 670-8.

5. Seckl MJ, Sebire NJ, Berkowitz RS. Gestational Trophoblastic Disease. Lancet 2010; 376: 717-29.

6. Lurain Jr. Gestational trophoblastic disease II: classification and management of gestational trophoblastic neoplasia. Am J Obstet Gynecol. 2011; 204(1): 11-8.

7. Mangili G, Lorusso D, Brown J, Pfisterer J, Massuger L, Vaughan M, et al. Trophoblastic disease review for diagnosis and management: a joint report from the International Society for the Study of Trophoblastic Disease, European Organisation for the Treatment of Trophoblastic Disease, and the Gynecologic Cancer InterGroup. Int J Gynecol Cancer 2014; 24( 9 supple 3): S 109-116

8. Alazzam M, Tidy J, Hancock BW, Osborne R, Lawrie TA. First-line chemotherapy in low-risk gestational trophoblastic neoplasia. Cochrane Database Syst Rev. 2012: CD007102.

9. Newlands ES, Bagshawe KD, Begent RH, Rustin GJ, Holden L. Results with the EMA/CO (etoposide, methotrexate, actinomycin D, cyclophosphamide, vincristine) regimen in high-risk gestational trophoblastic tumours, 1979 to 1989. Br J Obstet Gynecol 1991; 98: 550-7.

10. Hancock BW. Staging and classification of gestational trophoblastic disease. Best Pract Res Clin Obstet Gynecol 2003; 17: 869-83

11. Schmid P, Nagai Y, Agarwal R, Hancock B, Savage PM, Sebire NJ,et al. Prognostic markers and long-term outcome of placental-site trophoblastic tumours: a retrospective observational study. Lancet 2009; 374 ( 9807): 48-55

12. Yang J, Xiang Y, Wan X, Yang X. Recurrent gestational trophoblastic tumor: management and risk factors for 
recurrence. Gynecol Oncol 2006; 103: 587-90

13. Ngan HY, Seckl MJ, Berkowitz RS, Xiang Y, Golfier F, Sekharan PK, el al. Update on the diagnosis and management of gestational trophoblastic disease. Int J Gynecol Obstet 2015; 131: s123-6

14. Neubauer NL, Latif N, Kalakota K, Marymont M, Small W Jr, Schink JC, et al. Brain metastasis in gestational trophoblastic neoplasia: an update. J Reprod Med 2012; 57: 288-92

15. El-Helw LM, Hancock BW. Treatment of metastatic gestational trophoblastic neoplasia. Lancet Oncol 2007; 8: $715-24$

16. Azab MB, Pejovic MH, Theodore C, George M, Droz JP, Bellet D, et al. Prognostic factors in gestational trophoblastic tumors. A multivariate analysis. Cancer 1988; 62: 585-92

17. Kong Y, Yang J, Jiang F, Zhao J, Ren T, Li J, et al. Clinical characteristics and prognosis of ultra high-risk gestational trophoblastic neoplasia patients: A retrospective cohort study. Gynecol Oncol 2017; 146: 81-6

18. Bolze PA, Riedl C, Massardier J, Lotz JP, You B, Schott AM, et al. Mortality rate of gestational trophoblastic neoplasia with a FIGO score of $\geq 13$. Am J Obstet Gynecol 2016; 214: 390 e1-8

19. Suprasert P, Siriaree S, Manopunya M. Outcomes of Metastatic Gestational Trophoblastic Neoplasia: Fourteen Year Experience from a Northern Thailand Tertiary Care Center. Asian Pac J Cancer Prev. 2016; 17 (3): 1357-62

20. Savage P, Kelpanides I, Tuthill M, Short D, Seckl MJ. Brain metastases in gestational trophoblast neoplasia: an update on incidence, management and outcome. Gynecol Oncol 2015; 137: 73-6

21. Miller DS, Lurain JR. Classification and staging of gestational trophoblastic tumors. Obstet Gynecol Clin North Am. 1988 Sep;15(3):477-90.

22. Jiang F, Wan XR, Xu T, Feng FZ, Ren T, Yang JJ, et al. Evaluation and suggestions for improving the FIGO 2000 staging criteria for gestational trophoblastic neoplasia: A ten-year review of 1420 patients. Gynecol Oncol. 2018 Jun;149(3):539-544.

\section{c) (7) (8)}

This work is licensed under a Creative Commons AttributionNon Commercial 4.0 International License. 